\title{
An Easily-Assembled Soxhlet Extractor to Demonstrate Continuous Extraction
}

Kevin M. Jones*, Iain A. Smellie*, Iain L. J. Patterson

School of Chemistry, University of St Andrews, St Andrews, KY16 9ST

The construction of a Soxhlet extractor from readily available laboratory equipment is described. The apparatus can be used to demonstrate the concept of continuous extraction and was used in the isolation of trimyristin from nutmeg.

\section{GRAPHICAL ABSTRACT}

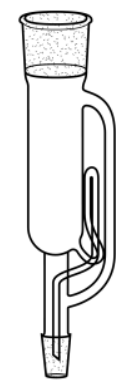

Soxhlet

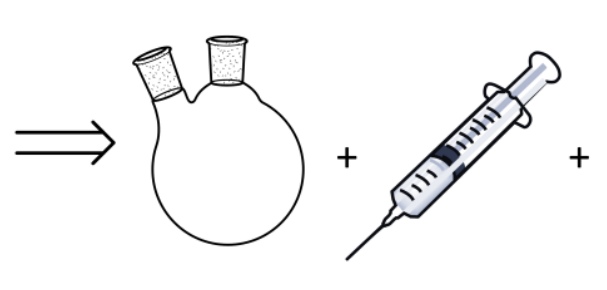

Flask



Tubing

\section{KEYWORDS}

High School / Introductory Chemistry, First-Year Undergraduate / General, Analytical Chemistry, Hands-On Learning / Manipulatives

The Soxhlet extractor is a classic piece of chemical apparatus ${ }^{1,2}$ used to extract sparingly soluble material from a solid sample. Several experiments based on this concept have been published in this Journal including the isolation of caffeine from beverage plants $^{3}$ and piperine from black pepper. ${ }^{4}$ Soxhlet extractors are intricate pieces of glassware making them both fragile and relatively expensive. The cost of such equipment 
can be restrictive, especially for schools and universities with limited budgets. ${ }^{5}$ In this paper we describe the construction of a Soxhlet extractor from readily-available equipment and demonstrate its use in the extraction of trimyristin from nutmeg. ${ }^{6}$

\section{AN EASILY-ASSEMBLED VARIANT} constructed from a $50 \mathrm{~mL}$ syringe barrel, glass vial filled with an appropriate coolant to act as a condenser and a siphon made from a length of tubing that serves to periodically empty the cavity allowing the solid to be extracted with fresh solvent. Material to be extracted is placed in a folded filter paper and inserted into the chamber. The extraction chamber is connected to a two-necked flask with a length of wide diameter plastic tubing and a $1 \mathrm{~mL}$ syringe barrel. Full details on how to construct the Soxhlet extractor and cross-sectional line drawings of a traditional, all-glass Soxhlet extractor compared to our easily-assembled variant can be found in the Supporting Information.

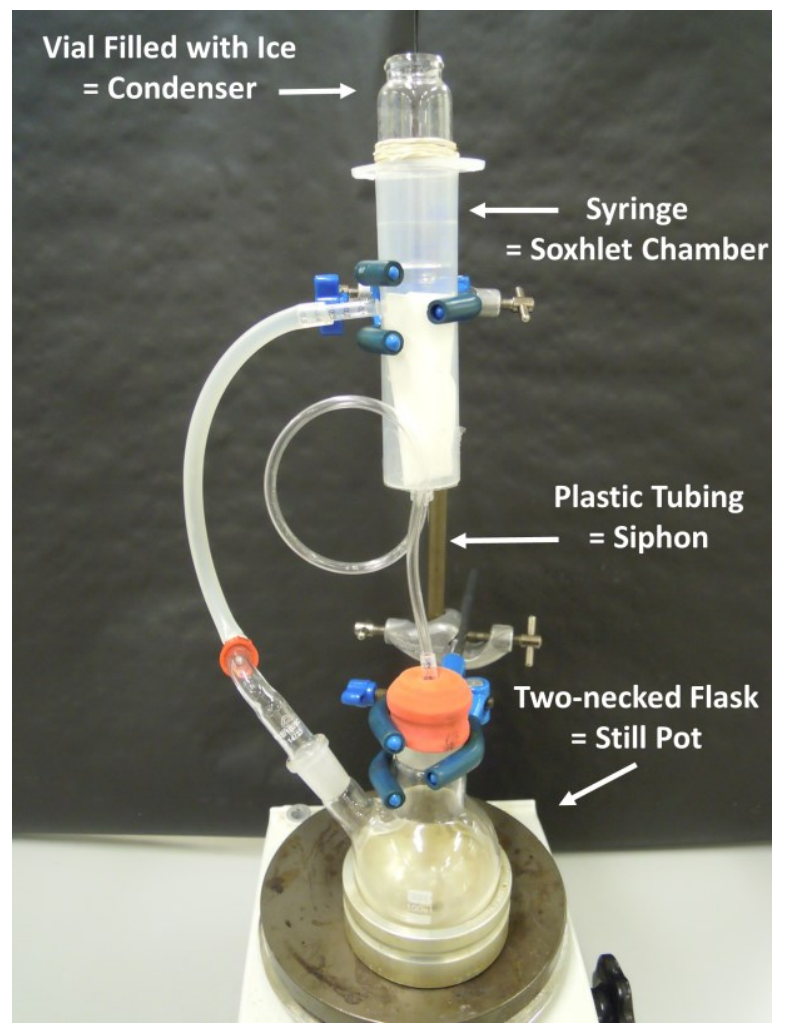


Figure 1.Assembled Soxhlet extractor consisting of a two-necked flask, syringe Soxhlet chamber containing material to be extracted, glass vial containing ice to act as a condenser and plastic tubing to serve as a siphon.

\section{EXPERIMENTAL}

To demonstrate the effectiveness of our apparatus, we examined the extraction of trimyristin ${ }^{7}$ from nutmeg following the procedure below:

Ground nutmeg $(3 \mathrm{~g})$ was placed in a thimble made from a folded filter paper and inserted into the Soxhlet chamber followed by a glass vial containing ice/water. ${ }^{8}$ Dichloromethane $(70 \mathrm{~mL})$ was added to the two-necked flask followed by 3 or 4 boiling chips. The flask was heated to reflux and allowed to operate for one hour. The coolant within the condenser was periodically replaced with a syringe/pipette to maintain a temperature capable of condensing the solvent vapor. After one hour the reaction vessel was removed from the heat and allowed to cool to room temperature. The boiling chips were removed by filtration and the resulting solution reduced to dryness on a rotary evaporator to afford yellow oil that solidified on cooling (1.2 g). The yellow solid was recrystallized from ethanol to afford a white solid ( $0.12 \mathrm{~g}, 4 \%$ based on mass of nutmeg, mp $\left.53^{\circ} \mathrm{C}\right)$. Full details can be found in the Supporting Information.

\section{SAFETY RECOMMENDATIONS}

Although in principle alternative solvents could be used in this version of the Soxhlet extractor, the authors strongly recommend that dichloromethane is used as the extraction solvent. Dichloromethane is non-flammable and has a low boiling point, which make it an ideal solvent for this demonstration.

\section{EXPERIMENT TESTING}

Next, we wished to determine whether our protocol could be reproduced in students' hands. Twenty undergraduate students carried out this experiment as part of an extracurricular lab course. Each student was provided with both written and video instructions and we were delighted to find that they were able to accurately reproduce 
the experiment with limited assistance. Students also provided feedback on minor problems they had encountered and how the instructions could be amended to circumvent these issues. As an example, several students used a particularly long piece of tubing to connect the round bottomed flask to the Soxhlet chamber. This was found to introduce dips in the tubing in which solvent could collect, hindering vapor reaching the Soxhlet chamber. This could be avoided by ensuring the tubing between the two vessels is as short as possible. A series of "construction tips" were added to the instructions to make users aware of these potential problems.

\section{HAZARDS}

Safety glasses, lab coats and gloves should be worn during all experimental work. The experiment should be fully risk-assessed before any practical work is undertaken. All experimental work should be carried out in a well-ventilated fume hood. All compounds should be treated as potentially hazardous. Dichloromethane can cause skin and serious eye irritation and may cause respiratory irritation and damage to organs. Ethanol is flammable and may cause irritation through contact or inhalation.

\section{CONCLUSION}

An easily-constructed Soxhlet extractor has been developed and tested through the extraction of trimyristin from nutmeg. Our hope is that this apparatus can be used to demonstrate the concept of continuous extraction to a wider range of students, especially younger students in a school environment.

\section{ASSOCIATED CONTENT}

Supporting Information

85 The Supporting Information is available on the CAS Publications website at DOI:

A document containing a parts list, instructions for making the Soxhlet extractor, a figure comparing traditional and easily-assembled variants and experimental details for the extraction and purification of trimyristin is provided. A video demonstrating the construction and operation of the Soxhlet extractor is also provided. 
AUTHOR INFORMATION

Corresponding Author

*E-mail: $\quad$ kj34@st-andrews.ac.uk

ias10@st-andrews.ac.uk

\section{ACKNOWLEDGMENTS}

We would like to thank the Engineering and Physical Sciences Research Council,

University of St Andrews, and the CRITICAT Centre for Doctoral Training for financial support [Grant code: EP/L016419/1].

\section{REFERENCES}

1. Jensen, W. B. The Origin of the Soxhlet Extractor. J. Chem. Educ. 2007, 84 (12), 1913-1914.

2. Royal Society of Chemistry. Chemistry World: Classic Kit-The Soxhlet Extractor. http://www.rsc.org/chemistryworld/Issues/2007/September/ClassicKitSoxhletE xtractor.asp (accessed 25th March 2017).

3. Adam, D. J.; Mainwaring, J. Soxhlet Extraction of Caffeine from Beverage Plants. J. Chem. Educ. 1996, 73 (12), 1171.

4. Epstein, W.W.; Netz, D.F.; Seidel, J. L. Isolation of Piperine from Black Pepper. J. Chem. Educ., 1993, 70 (7), 598-599.

5. Wesolowski, S. S.; Mulcahy, T.; Zafoni, C. M.; Wesolowsk, W. E. The Development of a Microscale Continuous Hot Solvent Extractor. J. Chem. Educ. 1999, 76 (8), 1116-1117.

6. Frank, F.; Roberts, T.; Snell, J.; Yates, C.; Collins, J. Trimyristin from Nutmeg. J. Chem. Educ. 1971, 48 (4), 255-256.

7. Although the easily-assembled Soxhlet extractor described can in principle be used for alternative extractions e.g. isolation of cinnamaldehyde from cinnamon, the authors recommend the isolation of trimyristin as the product can be easily purified by recrystallization making ideal for demonstrating the concept of continuous extraction.

8. Other coolant mixtures such as salt/ice and dry ice/acetone can also be used. 
